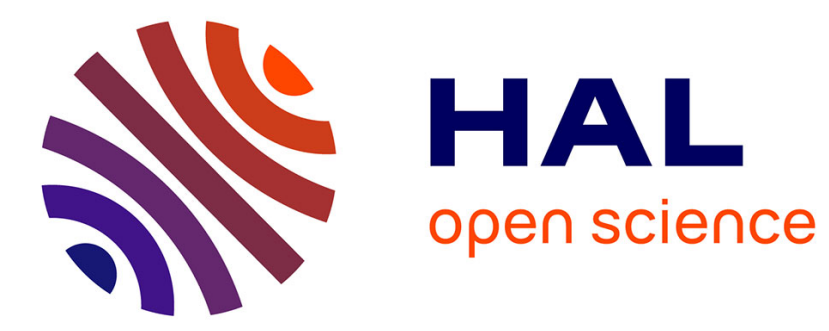

\title{
Objets provenant d'un cimetière protohistorique et gallo-romain à Lanslevillard (Savoie) \\ Jacques-Claude Courtois
}

\section{To cite this version:}

Jacques-Claude Courtois. Objets provenant d'un cimetière protohistorique et gallo-romain à Lanslevillard (Savoie). Gallia - Fouilles et monuments archéologiques en France métropolitaine, 1961, 19 (2), pp.245-248. 10.3406/galia.1961.2327 . hal-01926098

\section{HAL Id: hal-01926098 \\ https://hal.science/hal-01926098}

Submitted on 25 Feb 2020

HAL is a multi-disciplinary open access archive for the deposit and dissemination of scientific research documents, whether they are published or not. The documents may come from teaching and research institutions in France or abroad, or from public or private research centers.
L'archive ouverte pluridisciplinaire HAL, est destinée au dépôt et à la diffusion de documents scientifiques de niveau recherche, publiés ou non, émanant des établissements d'enseignement et de recherche français ou étrangers, des laboratoires publics ou privés.

\section{(ㅇ)(1) $\$$}

Distributed under a Creative Commons Attribution - NonCommercial - NoDerivatives| 4.0 


\section{DOCUMENTS COMMENTÉS}

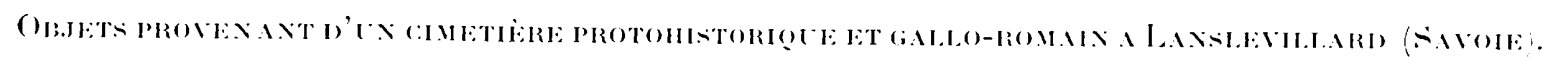

Ein septembre 19:2, M.M. J. Blanchard of (i. Connet. membres de la société Préhislorique Française. apprenaient lors d'une visile a Lanslevillard, canton de Lanslebourg. Saroit. en llaute-Maurienne. qu'on avait lrouve quelques tombes contenant de grands squelelles accompagnes de mobilier funéraire, au cours des travaux de reconstruction du village. presque entiorement delruit en 19-1.1. Ie cimetiere, déji connu par des lrouvalles antérieurese avait ele entame par les fondations de deux maisons. Selon M. Blanchard, il rest. certainement des parliess intacles qui pourront itre facilement fouillées aver lous les soins désirables (letlre du 3 novembre 195:) all Inrecteur des antiquilés préhistoriques: L'inrenteur des lombes est M. Marcelin Filliol, qui saura les objels en 19.19 ou 1950. Ces objets sont actuellement conserves dans les collerelions de la sociele d'histoire el d'archéologie de Maurienne. a saint-Jean-de-Maurienne, oi f'ai eu l'oceasion de les photographier, errace a l'obligeance of a l'amahilité du Chanoine J. Bellet, président de cette société savante.

selon les indications recueillies. les squelelles. tous de erande taille, de 1 m. 90 a 2 mitres (?). reposaient entre des dalles de schiste (lanzes) formant sarcophages. Ils élaient trés bien conservés. De la céramique aurail été lromée. outre les objets reproduits sur les pholographies, figures 1 a $i$; on remarquera surtol.l la belle série de fibules an forme de romelles. a ornementation émaillér et colorée. Celles-ri el

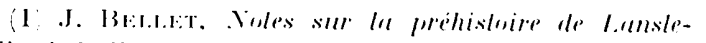
eillart. Belley. l!13!).

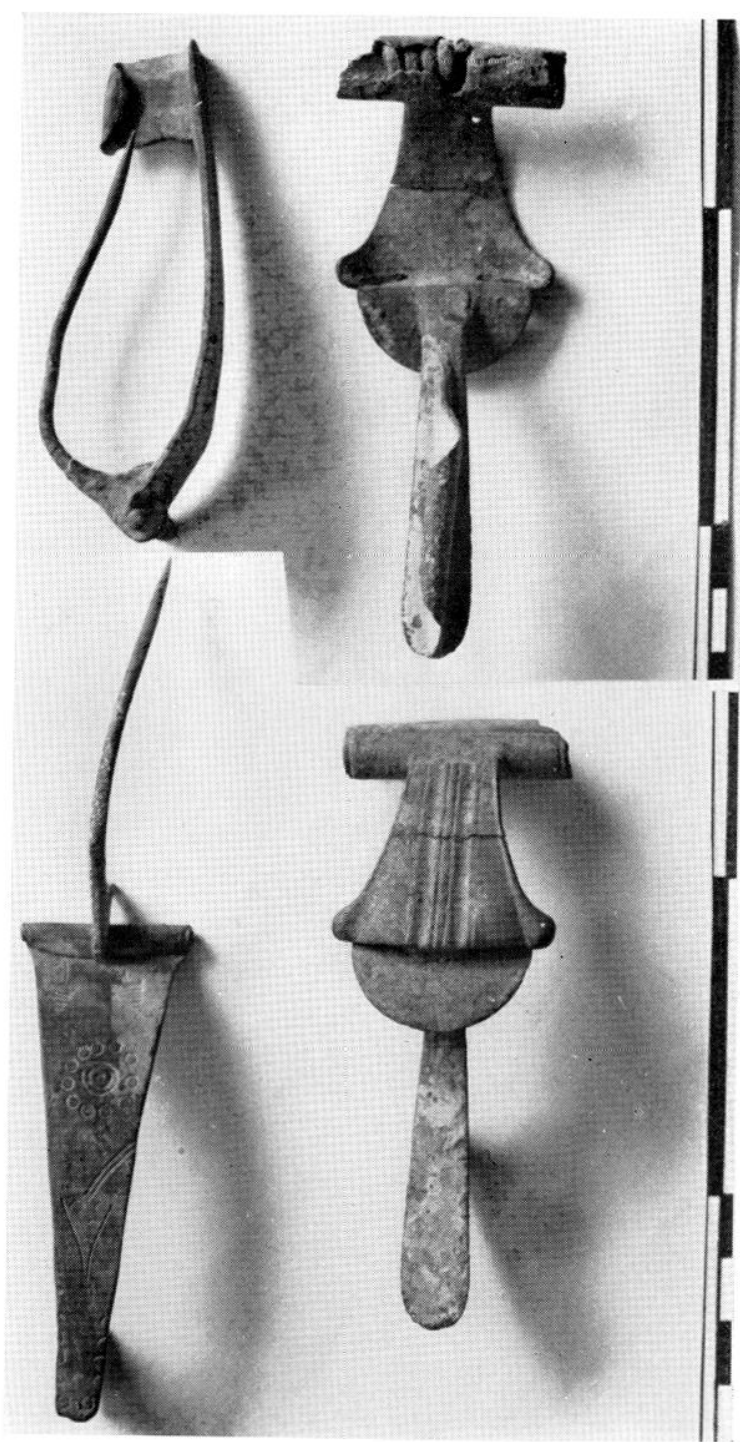

liier. 1. I.anslevilland sinuie.

Fibules on bromze. ri. liẹ. : e et 3 


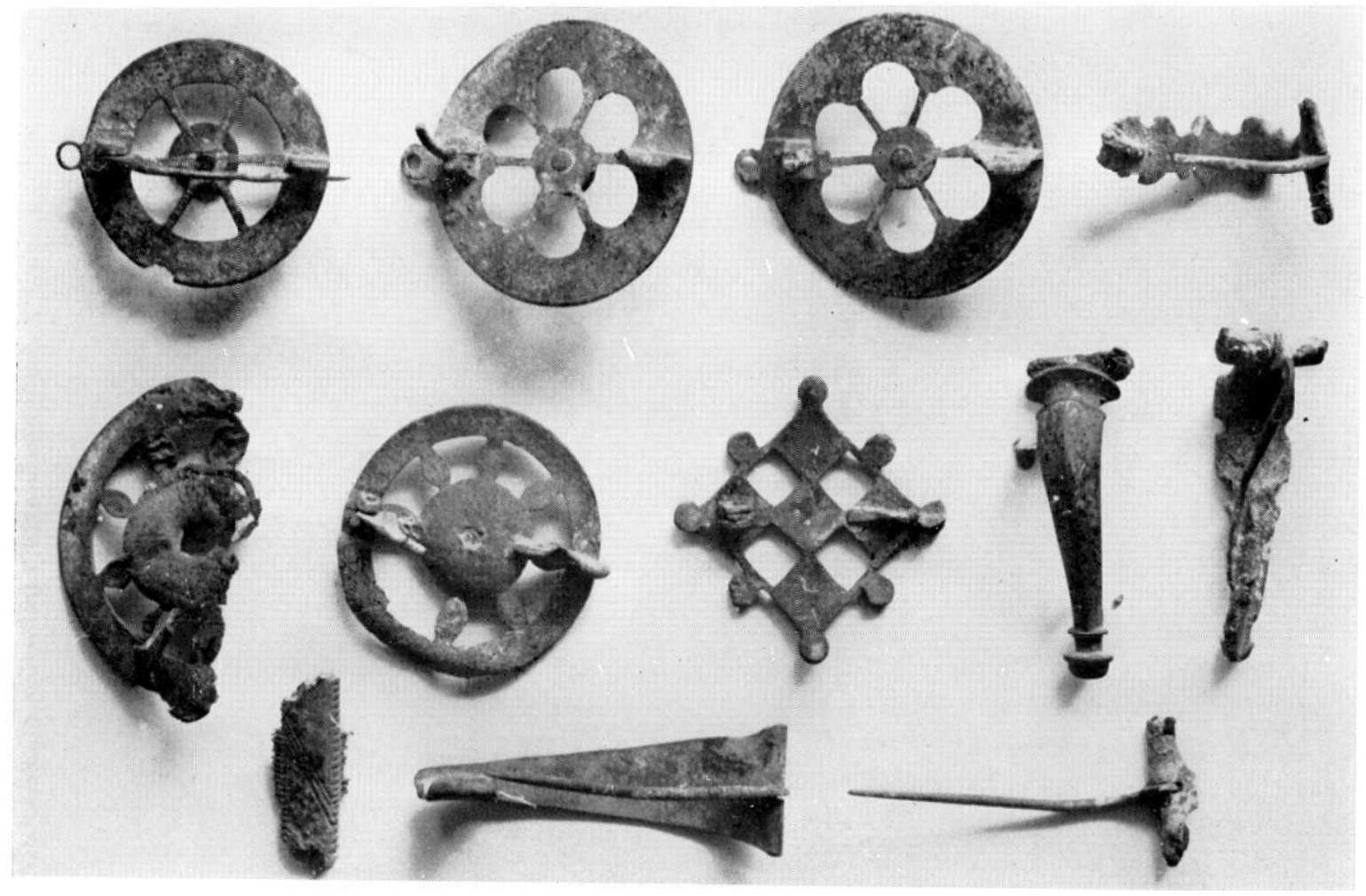

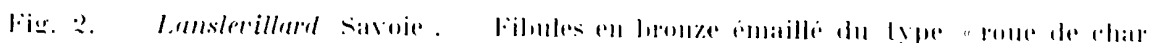

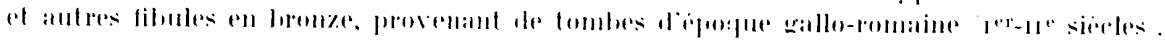

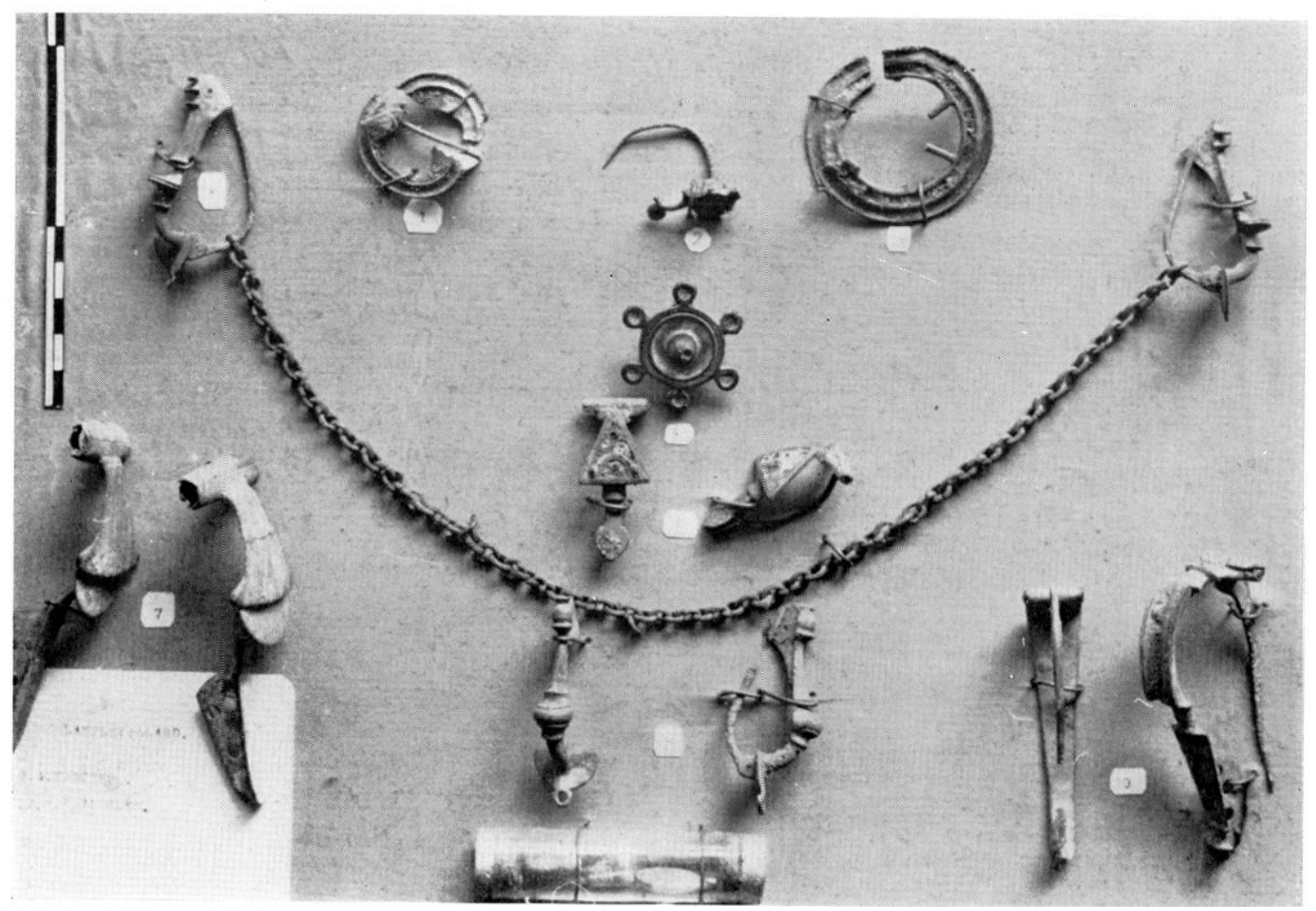

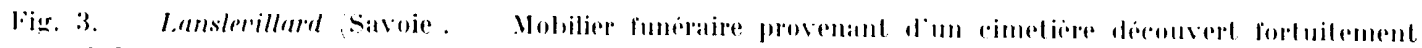

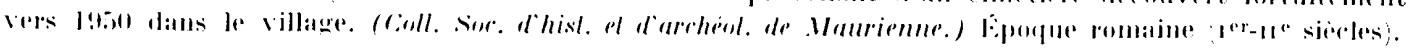




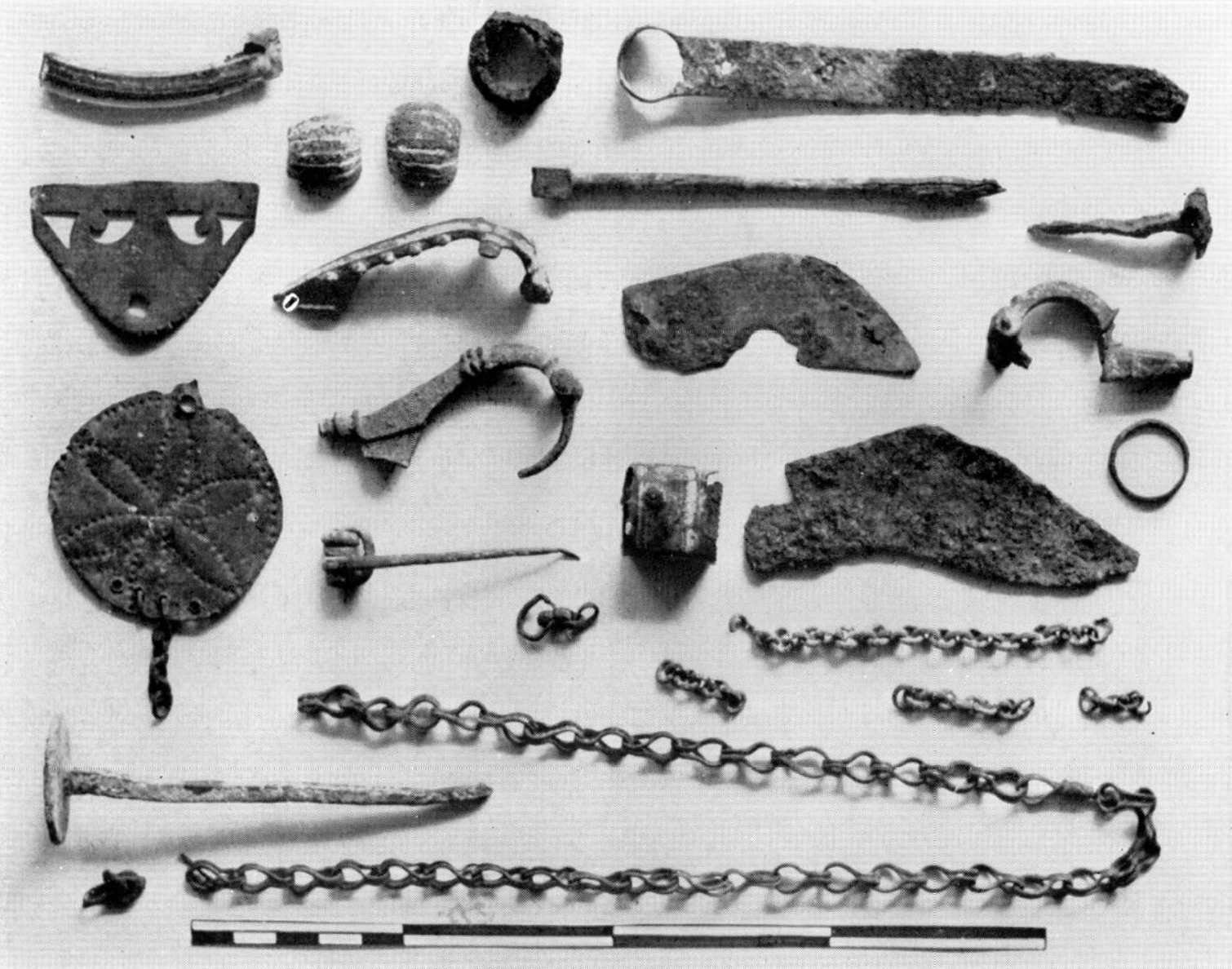

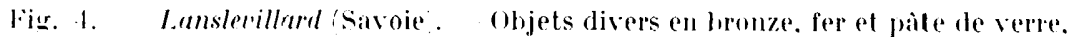

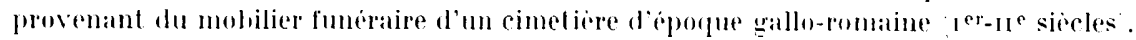

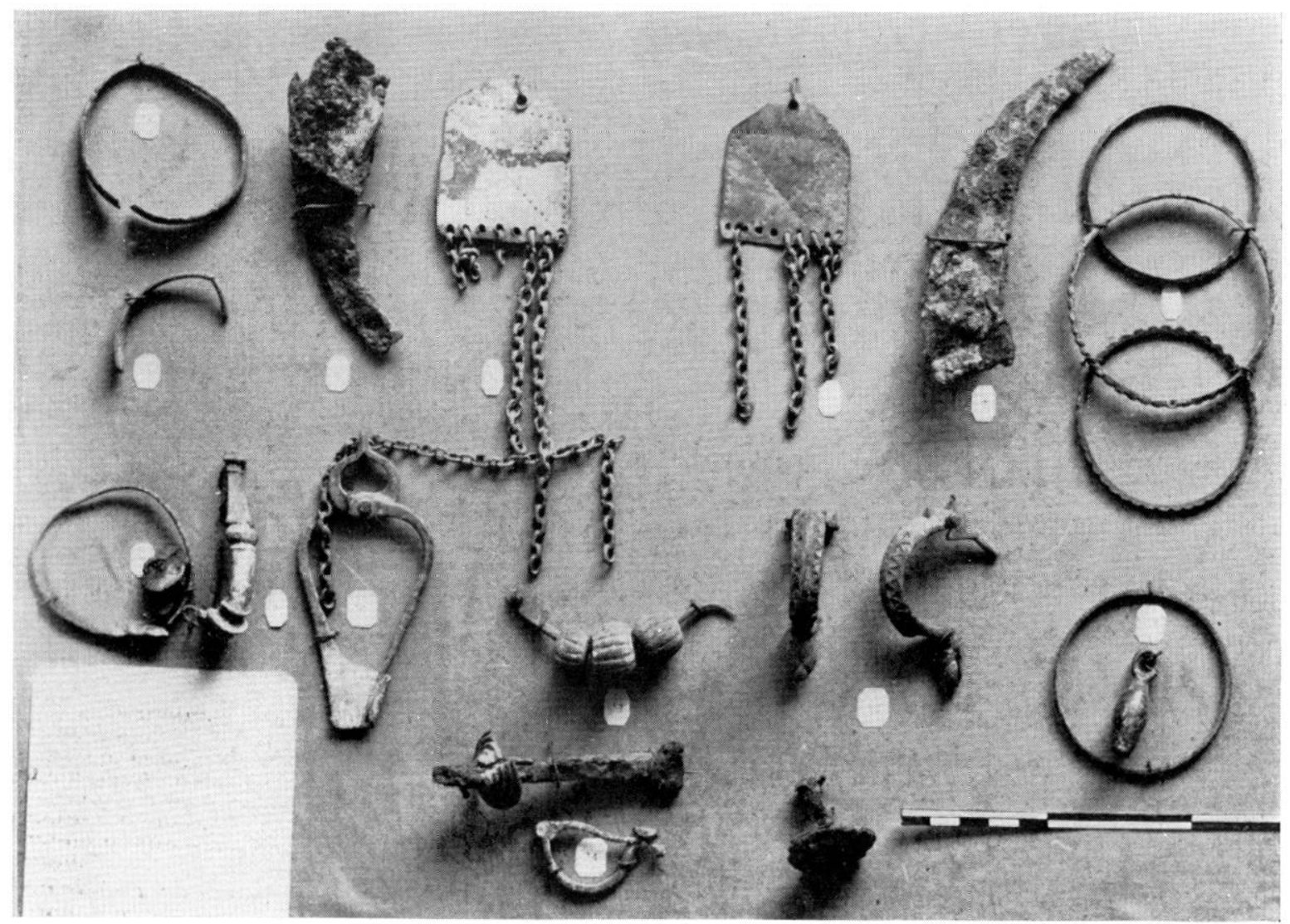

Fì. 5. L.anslerillard savoie. -- Objets du mobilier fumimire provenant des fouilles dun cimetire

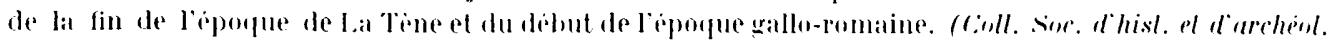
de Maurienne. Saint-Jean-te-Vaurienne. Savoie 
les aul res libules indiequenl une dale voisine du debul de l'bimpire cer-me siectes de nolre ire pour ce rimelieres qui liva auparavant des objels plus anciens de l'époque de la lione, nol amment des armilles filiformes el des fibules du lype an tenailles. ainsi que des pendeloques-crolales (fig. 5). Cependant. pour ne pas se meprendre sur la question relromologique. il convient de rappeler qu'un rertain conserva- lisme cullurel se manifeste a loules les cipoques dans res regions alpestres.

I rote des fibules. les objels romprennent des rouleaux en fer. des clous en fer. diverses chatonetles en bronze. quelques appliques in lóle de bronze, el des perles en pale de verre bleu, du lype courant dans loules les régions.

\section{J.-Cil. Colments.}

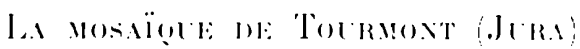

On a découvert. il y a environ 20) ans, en 1711 ou 1717 pres d'un village du Jura. Toumont arrondissement de Poligny au Cord de Lons-le-saunier au lieu-dil Les C.hambrelles ou Eskabrege. a list mitres ensiron al XordOuest du village. sur le bord de la roule de bole. une mosaïgue romaine qui allia l'allen-

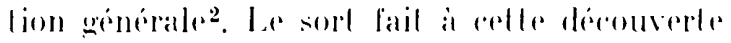
a de un pen plus favorable que dans bien d'alleres ras du memes gente. Ce pavement. enfoui une premiere fois par l'inventeur. le lermier Fleur de Tourmonl. pour le proléger eontre les delépioralions par des visileurs indiserels. fut degage a noureaul a l'oceasion d'une visite de l'historien l)unod de Ciharnage. de Besancon, yui vinl le voir en ortobre 17.6103. Reste probablement a ciel ouvert justu'en seplembre 17\%), il ful alors lobjel d'une autre visite de personnages d'une certaine importance, de l'historien de Poligny. Fr.-F. Chevalier, et du Marquis de Montrichardt. Gràce à ces deux visiles.

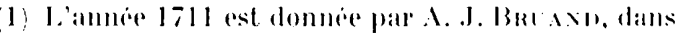
som rapport de la decouverle, paru dans l'Almuaire de

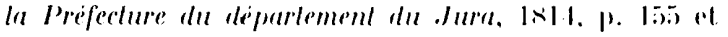

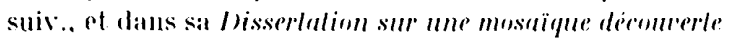
pres de la rille de Poligny. Patris, lisli, p. i, alors que

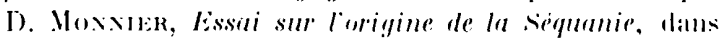
Almanarh historique el agronmmique de la l'refeclure du .Jura pour lannée 1819, p. 191 et suis., indiepue 1717.

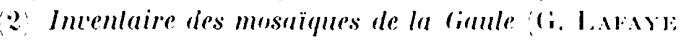

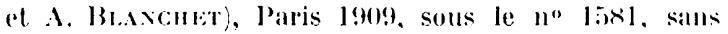
illustration. Portera le no 316 dans. H. Srters, Recueil gencral des musaïques de la Ciaule, t. I. 3. a jaraitre.

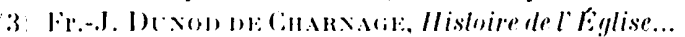
ar Besancon. I I. 17501, 1. 35: a a 359.

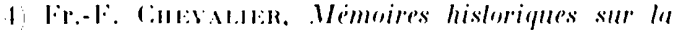

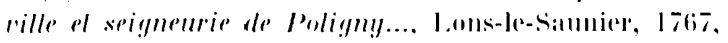
p. I.X.X i $1: 11$. nous an possedons deux descriptions assez délaillées, ol qui plus est. une gravure que Caylus a publice au lome IV de son Recueil dranliquiles. d'apres un dessin lransmis par le marequis de Montrichard the. 1 Par la suite el loujours dans le meme but de protection contre l'ondommagement par les visilems, la mosaïyue lut entouie de nomrau. I en reroire 1). Bomniere on l'aurail dégagée encore une fois en list: peut-ible dans l'intention de l'enlever el de la placer dans le musée fonde alors a lons-le-samnier. Nais on en resta aux projets. Ce documenl remarquable de l'arl romain en Gaule ne sortil pas de lerre of y est ancore de nos jours. 1 la fin du siede passe un homonyme de 1). Momnier. Pierre Momnier, fit ampagne. pour une fouille mélhodique. sans résullal?

Vous iqnorons dans quel étal se troure aujourd'hui celle mosaïque, qui, a en croire les témoins orulaires, élail parfailement conservere all moment de sa découverte. Les mulliples operalions de degagement el d'enfouissement, les prélexements de cubes par les visileurs. noul cerles pas manqué de la delériorer, mais il semblemail qu'une partie imporlanle en existe encore. It serait possible de la mellre au jour.

le prime abord l'elude d'un parement qui ne nous est eonmu que par des descriptions et par une gravure du xime siocle parait offrir

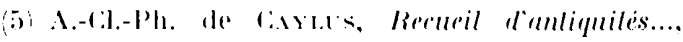
IV. Paris, 1761, pl. tiNill.

(i) lone. ril.

7 l. Moxwlis. la mostüque gallo-romaine de Tourmunl pres de Polign!y (.lura). daus Soles darl

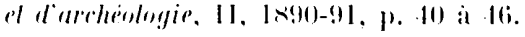

